\title{
GLOBAL NON-NEGATIVE CONTROLLABILITY OF THE SEMILINEAR PARABOLIC EQUATION GOVERNED BY BILINEAR CONTROL
}

\begin{abstract}
Alexander Y. Khapalov ${ }^{1}$
Abstract. We study the global approximate controllability of the one dimensional semilinear convection-diffusion-reaction equation governed in a bounded domain via the coefficient (bilinear control) in the additive reaction term. Clearly, even in the linear case, due to the maximum principle, such system is not globally or locally controllable in any reasonable linear space. It is also well known that for the superlinear terms admitting a power growth at infinity the global approximate controllability by traditional additive controls of localized support is out of question. However, we will show that a system like that can be steered in $L^{2}(0,1)$ from any non-negative nonzero initial state into any neighborhood of any desirable non-negative target state by at most three static ( $x$-dependent only) above-mentioned bilinear controls, applied subsequently in time, while only one such control is needed in the linear case.
\end{abstract}

Mathematics Subject Classification. 93, 35.

Received January 17, 2001. Revised May 21, September 8 and October 1, 2001.

\section{INTRODUCTION}

\subsection{Motivation}

In the mathematical models of controlled distributed parameter processes boundary and interior locally distributed or lumped controls are typically used. They enter the model as additive terms (note that the boundary controls can be modeled by additive Dirac functions) and have localized support. In terms of applications it appears that these controls can adequately model only those processes that do not change their principal physical characteristics due to the control action. They rather describe the affect of various external ("alien") forces on the process at hand. This "limitation", however, excludes numerous new and not quite new technologies, such as, for example, the chemical reactions, controlled by "catalysts", and "smart materials", which are able to change their principal parameters under certain conditions. Moreover, in the technical aspect, it turns out that the additive controls may not be effective when dealing with highly nonlinear problems.

In this paper we attempt to address the just-outlined issues in the context of approximate controllability of the semilinear parabolic equation governed via a coefficient (bilinear control) in its additive reaction term. Such (bilinear) control can change at least some of the principal parameters of the process at hand, for example, the rate of a chemical (or chain) reaction, which can be achieved by using various catalysts and/or by the "speed" at which the reaction ingredients are mixed.

Keywords and phrases: Semilinear parabolic equation, global approximate controllability, bilinear control.

${ }^{1}$ Department of Pure and Applied Mathematics, Washington State University, Pullman, WA 99164-3113, U.S.A.;

e-mail: khapala@wsu.edu 


\subsection{Problem formulation}

Let us consider the following Dirichlet boundary problem, governed in a bounded domain $(0,1)$ by the bilinear control $\alpha \in L^{\infty}\left(Q_{T}\right)$ :

$$
\begin{aligned}
& u_{t}=u_{x x}+\alpha u-f\left(x, t, u, u_{x}\right) \quad \text { in } \quad Q_{T}=(0,1) \times(0, T), \\
& u(0, t)=u(1, t)=0, \quad t \in(0, T),\left.\quad u\right|_{t=0}=u_{0} \in L^{2}(0,1) .
\end{aligned}
$$

Given $T>0$, we further assume that $f(x, t, u, p)$ is Lebesgue's measurable in $x, t, u, p$, and continuous in $u, p$ for almost all $(x, t) \in Q_{T}$, and is such that

$$
\begin{gathered}
|f(x, t, u, p)| \leq \beta|u|^{r_{1}}+\beta|p|^{r_{2}} \quad \text { a.e. in } Q_{T} \text { for } u, p \in R, \\
\int_{\Omega} f\left(x, t, \phi, \phi_{x}\right) \phi \mathrm{d} x \geq 0 \quad \forall \phi \in H_{0}^{1}(0,1),
\end{gathered}
$$

where $\beta>0$, and

$$
r_{1} \in(1,5), \quad r_{2} \in(1,5 / 3) .
$$

A simple example of a function $f$ satisfying conditions (1.2a-1.2c) is $f(u)=u^{3}$. Note that (1.2a) and (1.2c) mean that $|f|$ is bounded above by a strictly superlinear function in $u$ and $p$ near the origin as well (see also Rem. 4.1 below in this respect).

Here and below we use the standard notations for Sobolev spaces such as $H_{0}^{1,0}\left(Q_{T}\right)=L^{2}\left(0, T ; H_{0}^{1}(0,1)\right)=$ $\left\{\phi \mid \phi, \phi_{x} \in L^{2}\left(Q_{T}\right), \phi(0, t)=\phi(1, t)=0\right\}$ and $H_{0}^{1}(\Omega)=\left\{\phi \mid \phi, \phi_{x} \in L^{2}(0,1), \phi(0)=\phi(1)=0\right\}$.

We refer, e.g., to [20] (p. 466), where it was shown that system $(1.1,1.2 \mathrm{a}-1.2 \mathrm{c})$ admits at least one generalized solution in $C\left([0, T] ; L^{2}(0,1)\right) \bigcap H_{0}^{1,0}\left(Q_{T}\right) \cap L^{6}\left(Q_{T}\right)$, but its uniqueness is not guaranteed.

In the context of the heat (mass)-transfer $\alpha$ is proportional to the heat-transfer (or mass-transfer) coefficient, which depends on the environment, the substance at hand, and its surface area. If the heat (mass)-transfer involves fluids (air), $\alpha$ also depends on the speed of the fluid. (The latter can be controlled in some applications by the artificially induced magnetic field.) Alternatively, the surface area can be changed when the substance at hand is a polymer (e.g., a planar array of gel fibers can be controlled to maximize the surface area exposed to the surrounding fluid). Also, we refer to the so-called "extended" surface applications (fins, pins, studs, etc.) when one wishes to increase/decrease the exchange between source and an ambient fluid.

In this paper we are concerned with the issue of approximate controllability of system (1.1, 1.2a-1.2c). Note that, even in the case when $f=0$, that is, when (1.1) becomes the linear boundary problem

$$
\begin{gathered}
y_{t}=y_{x x}+\alpha y \quad \text { in } \quad Q_{T}=(0,1) \times(0, T), \\
y(0, t)=y(1, t)=0, \quad t \in(0, T),\left.\quad y\right|_{t=0}=y_{0} \in L^{2}(0,1),
\end{gathered}
$$

its solution depends highly nonlinearly on $\alpha$, which makes the associated controllability problem nonlinear as well.

Let us recall that, in its general form, it is said that the system at hand is globally approximately controllable in the given (linear phase-) space $H$ at time $T>0$ if it can be steered from any initial state in $H$ into any neighborhood of any desirable target state in $H$ at time $T$, by selecting a suitable available (traditionally, linear additive) control.

However, it is not unnatural to expect that the use of bilinear controls and/or the presence of superlinear nonlinearity can give rise to certain conceptual modifications of this property. We refer in this respect to the early pioneering work [5] by Ball et al. on controllability of the abstract infinite dimensional bilinear system. In [5], the global approximate controllability of the rod equation $u_{t t}+u_{x x x x}+k(t) u_{x x}=0$ with hinged ends and of the wave equation $u_{t t}-u_{x x}+k(t) u=0$ with Dirichlet boundary conditions, where $k$ is control (the axial load), was shown making use of the nonharmonic Fourier series approach under the additional (nontraditional) 
assumption that all the modes in the initial data are active. (We also refer to [19] further exploring the ideas of [5] in the context of simultaneous control of the rod equation and Schrödinger equation.)

Let us discuss now some of the inherent difficulties arising in the context of bilinear controllability of parabolic pde's like $(1.1,1.3)$ and suggest a new definition of approximate controllability, which seems to be quite natural and consistent with potential applications.

First of all, whenever the zero state is a fixed point for the system at hand, it is immediately clear that such a system is not globally controllable from $y_{0}=0$ and its exact null controllability is out of question as well. Furthermore, even if $y_{0} \neq 0$ and $f=0$ the resulting seemingly "simpler" linear system (1.3) still cannot be approximately controllable by bilinear control $\alpha$ in any "reasonable" linear space. Indeed, e.g., if $y_{0}(x)$ is nonnegative, then, the maximum principle implies that $y(x, t)$ must remain non-negative for all $t>0$, regardless of the choice of $\alpha \in L^{\infty}\left(Q_{T}\right)$. In other words, one is unable to reach any of the "negative" (or, alternatively, positive) target states from a non-negative (or, alternatively, non-positive) initial state.

Accordingly, it seems of interest to study the approximate controllability of $(1.1,1.3)$ while dealing exclusively with non-negative initial and target states. In terms of applications, such modification is very natural. For example, if (1.3) describes a diffusion process with $y(x, t)$ being the concentration of a substance at point $x$ at time $t$, then, of course, it cannot accept the negative values. Alternatively, if (1.3) describes a heat-transfer process, with $y(x, t)$ being the temperature at point $x$ at time $t$, then it is also natural to assume that the temperature cannot fall below certain level (one may also recall the concept of absolute zero).

Secondly, the fact that the bilinear control $\alpha$ in $(1.1,1.3)$ is a coefficient opens certain possibilities to steer the system at hand to the desirable target state by creating suitable "drift" motions towards it. Namely, by making this target state a new equilibrium. This can be achieved by static (time-independent) bilinear controls, i.e., of a much simpler structure to implement, compared to, e.g., the traditional $x$ - and $t$-dependent locally distributed $L^{2}\left(Q_{T}\right)$-controls. On the other hand, such static controls will not allow us to comply with the "traditional approximate controllability" requirement that the control time should be the same for any pair of the initial and target states.

Thirdly, the above-cited classical definition of approximate controllability becomes ill-posed (and rather "questionable" in terms of applications) when the system at hand admits multiple solutions.

We summarize the above discussion by introducing the following definition:

Definition 1.1. We will say that system $(1.1,1.2 \mathrm{a}-1.2 \mathrm{c})$, generally admitting multiple solutions, is "non-negatively" globally approximately controllable in $L^{2}(\Omega)$ if for every $\varepsilon>0$ and non-negative $u_{0}, u_{d} \in$

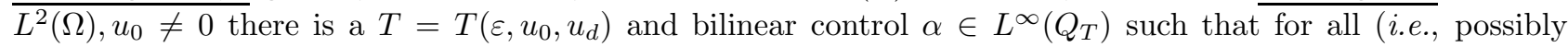
multiple) solutions of $(1.1,1.2 \mathrm{a}-1.2 \mathrm{c})$, corresponding to it,

$$
\left\|u(\cdot, T)-u_{d}\right\|_{L^{2}(\Omega)} \leq \varepsilon
$$

\subsection{Main results}

Our first result deals with the linear boundary problem (1.3).

Theorem 1.1. System (1.3) is "non-negatively" approximately controllable in $L^{2}(0,1)$ in the sense of Definition 1.1 by means of static controls $\alpha=\alpha(x), \alpha \in L^{\infty}(0,1)$ only. Moreover, the corresponding solution to (1.3) remains non-negative at all times. (No multiple solutions in this case.)

Theorem 1.2. System $(1.1,1.2 a-1.2 c)$ is "non-negatively" approximately controllable in $L^{2}(\Omega)$ in the sense of Definition 1.1. The corresponding steering can be achieved by subsequent applying of three suitable static bilinear controls. 
Corollary 1.3. The condition that the initial states $y_{0}$ and $u_{0}$ in Theorems 1.1 and 1.2 are nonzero and nonnegative can be replaced with the following more general assumptions:

$$
\int_{0}^{1} y_{0} y_{d} \mathrm{~d} x>0, \quad \int_{0}^{1} u_{0} u_{d} \mathrm{~d} x>0 \text {. }
$$

(However, $y(x, t)$ may now accept both negative and positive values during the steering process.)

Corollary 1.3 extends the results of Theorems 1.1 and 1.2 to a wider set of initial states (i.e., not necessarily non-negative), but, formally, it excludes the zero target states $y_{d}=u_{d}=0$. However, we show below in the first step of the proof of Theorem 1.2 that the zero target state can approximately be reached arbitrarily fast from any initial state in $L^{2}(0,1)$.

The remainder of the paper is organized as follows. Theorem 1.1 is proven in Section 2. Section 3 deals with some preliminary estimates, further used in Section 4 to prove Theorem 1.2. In Section 4 we discuss our main results, some open questions, and related additional references.

Remark 1.1. It is noteworthy that the global approximate controllability of the semilinear heat equation with superlinear power-like terms as in $(1.1,1.2 \mathrm{a}-1.2 \mathrm{c})$ is not possible by means of additive locally distributed controls [11,12]. More precisely, solutions to such an equation remain uniformly bounded outside the control support, regardless of the magnitude of control applied. (Hence, the non-negative global controllability in the sense of Def. 1.1 is out of question as well.) On the positive global controllability results for semilinear parabolic equations with superlinear terms and additive controls of localized support we refer to $[1,6,8,9,13-15]$ (see also the discussion on [16] in the beginning of Sect. 4).

\section{Proof of Theorem 1.1}

Our central idea here is to try to select $\alpha=\alpha(x)$ in such a way that the target state $y_{d}$ (or its "close" approximation) becomes co-linear to the first (non-negative) eigenfunction for the diffusion-reaction term $y_{x x}+$ $\alpha(x) y$ in (1.3), which is then approached by the corresponding trajectory of (1.3) as $t$ increases.

\subsection{Preliminaries}

Denote by $\lambda_{k}$ and $\omega_{k}(x), k=1, \ldots$ respectively the eigenvalues and orthonormalized in $L^{2}(0,1)$ eigenfunctions of the spectral problem $\omega_{x x}+\alpha(x) \omega=\lambda \omega, \omega \in H_{0}^{1}(0,1)$ (which, in fact, is a linear ode). It is known that

$$
\|\alpha\|_{L^{\infty}(0,1)} \geq \lambda_{1}>\lambda_{2}>\ldots,
$$

and $\lambda_{k} \rightarrow-\infty$ as $k$ increases. The unique solution to $(1.3)$ in $C\left([0, T] ; L^{2}(0,1)\right) \bigcap H_{0}^{1,0}\left(Q_{T}\right)$ admits the following representation:

$$
y(x, t)=\sum_{k=1}^{\infty} \mathrm{e}^{\lambda_{k} t}\left(\int_{0}^{1} y_{0}(r) \omega_{k}(r) \mathrm{d} r\right) \omega_{k}(x)
$$

Note that, for the given $\alpha$, we can endow the space $H_{0}^{1}(0,1)$ with the norm

$$
\|\omega\|_{H_{0}^{1}(0,1)}=\left(\int_{0}^{1}\left(\omega_{x}^{2}+(-\alpha(x)+c) \omega^{2}(x)\right) \mathrm{d} x\right)^{1 / 2}
$$


where $c$ is any positive constants exceeding $\|\alpha\|_{L^{\infty}(0,1)}$. Then,

$$
\left.\left\|\omega_{k}\right\|_{H_{0}^{1}(0,1)}=\left(\left(c-\lambda_{k}\right) \int_{0}^{1} \omega_{k}^{2}(x)\right) \mathrm{d} x\right)^{1 / 2}
$$

and

$$
\begin{gathered}
\|y(\cdot, t)\|_{L^{2}(0,1)} \leq \mathrm{e}^{\lambda_{1} t}\left\|y_{0}\right\|_{L^{2}(0,1)}, \quad t \geq 0, \\
\|y(\cdot, t)\|_{C[0,1]} \leq C_{*}\|y(\cdot, t)\|_{H_{0}^{1}(0,1)} \leq C(t)\left\|y_{0}\right\|_{L^{2}(0,1)}, \quad t>0,
\end{gathered}
$$

where $C_{*}$ is a positive constant associated with the continuous embedding $H_{0}^{1}(0,1) \subset C[0,1]$ and the function $C(t), t>0$ is nondecreasing. (Both $C_{*}$ and $C(t)$ depend on $\alpha$.)

\subsection{Proof of Theorem $\mathbf{1 . 1}$}

Our plan of the proof of Theorem 1.1 is as follows:

(i) We intend to show that for "almost any" given non-negative target state $y_{d} \in L^{2}(0,1)$ we can select an $\alpha_{*}(x)$ such that $y_{d} /\left\|y_{d}\right\|_{L^{2}(0,1)}$ becomes the first eigenfunction for (1.3), associated with the largest eigenvalue in the representation (2.2). In other words, in (2.2),

$$
\omega_{1}(x)=\frac{y_{d}(x)}{\left\|y_{d}\right\|_{L^{2}(0,1)}} .
$$

(ii) Then we will show that the actual control can be selected as

$$
\alpha(x)=\alpha_{*}(x)+a,
$$

where $a$ is a constant chosen so that the first term in the corresponding representation (2.2) converges to $y_{d}$, while the remainder of the series converges to zero as $t$ increases. Note that adding $a$ in the above does shift the eigenvalues corresponding to $\alpha_{*}$ (denote them by $\left\{\lambda_{k}\right\}_{k=1}^{\infty}$ now) from $\lambda_{k}$ to $\lambda_{k}+a$, but the eigenfunctions remain the same for $\alpha_{*}$ and $\alpha_{*}+a$.

Step 1. Maximum principle. Let us recall first that according to the generalized maximum principle

$$
0 \leq y(x, t) \leq \mathrm{e}^{b t}\left\|y_{0}\right\|_{L^{\infty}(0,1)}, \quad b=\|\alpha\|_{L^{\infty}(0,1)}, \quad t>0,
$$

whenever $y_{0} \in L^{\infty}(0,1)$ and is a.e. non-negative. Indeed, if $\alpha(x) \leq 0$ this is true with $b=0$ [16] (in this case (2.6) becomes the standard generalized maximum principle). Otherwise, equation (2.6) follows from the maximum principle applied to the function $z(x, t)=\mathrm{e}^{-b t} y(x, t)$, satisfying

$$
\begin{gathered}
z_{t}=z_{x x}+(\alpha(x)-b) z \text { in } Q_{T} \\
z(0, t)=z(1, t)=0, \quad t \in(0, T),\left.\quad z\right|_{t=0}=y_{0} \in L^{\infty}(0,1)
\end{gathered}
$$

with $\alpha(x)-b \leq 0$.

Note now that any non-negative $y_{0} \in L^{2}(0,1)$ can be approximated in $L^{2}(0,1)$ by a sequence of non-negative $y_{0 k} \in C[0,1]$. Due to $(2.4)$ the solutions to $(1.3)$ corresponding to $y_{0}$ and $y_{0 k}$ converge to each other in $C[0,1]$ 
for every positive $t$. Hence, in view of (2.6), we also have

$$
y(x, t) \geq 0 \text { in }(0,1) \times(0, \infty)
$$

whenever $y_{0}$ is non-negative element of $L^{2}(0,1)$.

Step 2. To prove Theorem 1.1, it is sufficient to consider any set of non-negative target states $y_{d}$ which is dense in the set of all non-negative elements of $L^{2}(0,1)$. To this end, we will consider only (a) nonzero non-negative continuously differentiable functions $y_{d}=y_{d}(x), x \in[0,1]$ that (b) vanish at $x=0,1$ and (c) whose second derivatives are piecewise continuous with finitely many discontinuities of the first kind (hence, $\left.y_{d} \in H^{2}(0,1) \cap H_{0}^{1}(0,1)\right)$ such that $(\mathrm{d})$

$$
y_{d}(x)>0 \text { in }(0,1) \text { and } \frac{y_{d x x}}{y_{d}} \in L^{\infty}(0,1) .
$$

To ensure the last condition in (2.8) it is sufficient to select $y_{d}$ that, in addition to the above, is linear near the endpoints $x=0,1$. This would guarantee that $y_{d x x}=0$ near $x=0,1$, while elsewhere, due to the first condition in (2.8), the denominator in (2.8) is strictly separated from 0.

Let us show that any non-negative element $g \in L^{2}(0,1)$ can indeed be approximated in this space by a sequence of functions described in the above.

Firstly, note that without loss of generality, we can assume that $g(x) \geq c>0$ for almost all $x \in(0,1)$ (since any non-negative function $g$ can be approximated, e.g., by a sequence of positive functions $g(x)+1 / k$, where $k \rightarrow \infty)$.

Secondly, recall that any $g \in L^{2}(0,1)$, positive a.e. in $(0,1)$ as described in the above, can be approximated in this space by a sequence of piecewise constant positive functions $g_{k}(x)$ with possible jumps at $x_{j}=j / k, j=$ $1, \ldots, k, x_{0}=0, x_{k}=1$, where

$$
g_{k}(x)=\frac{1}{k} \int_{x_{j-1}}^{x_{j}} g(x) \mathrm{d} x, \quad x \in\left[x_{j-1}, x_{j}\right), \quad j=1, \ldots, k .
$$

Note that $g_{k}(x)$ are strictly separated from zero in $(0,1)$.

Thirdly, each of such piecewise constant functions can in turn be approximated in $L^{2}(0,1)$ by continuous piecewise linear functions that vanish at $x=0,1$ and everywhere else are strictly positive, whose graphs, accordingly, do not have vertical pieces. (Namely, for that one just needs, e.g., to connect the graphs of the former functions by the pieces of straight lines near the discontinuity points.)

Finally, each of these broken lines can be "smoothened" at the corners, e.g., by using pieces of circles of "sufficiently small" radia with centers located on the bisectors of the angles generated by the corresponding adjacent straight lines of the graphs (so that these lines are tangent to the associated circles). These "smoothened" lines are the graphs of the functions satisfying all the conditions (a-d) described in the above and approximate in $L^{2}(0,1)$ the above-constructed broken lines (and, hence, eventually an arbitrarily selected non-negative $\left.g \in L^{2}(0,1)\right)$ as the above-mentioned radia tend to zero.

Step 3. Select any non-negative nonzero $y_{0} \in L^{2}(0,1)$ and any $y_{d}$ as described in the above. Set

$$
\alpha_{*}(x)=-\frac{y_{d x x}(x)}{y_{d}(x)}, \quad x \in(0,1) .
$$

Note that $\alpha_{*}(x)$ is not identically zero in $L^{\infty}(0,1)$ (otherwise, $y_{d} \equiv 0$.) The eigenvalues associated with this $\alpha_{*}$ we further denote by $\left\{\lambda_{k}\right\}_{k=1}^{\infty}$.

(2.9) means that the function

$$
\frac{y_{d}(x)}{\left\|y_{d}\right\|_{L^{2}(0,1)}}
$$


is an eigenfunction for (1.3) with $\alpha=\alpha_{*}$, say

$$
\frac{y_{d}(x)}{\left\|y_{d}\right\|_{L^{2}(0,1)}}=\omega_{k *}(x)
$$

associated with the zero eigenvalue $\lambda_{k *}=0$.

Note now that, since all the eigenfunctions are orthogonal in $L^{2}(0,1)$, that is,

$$
\int_{0}^{1} \omega_{k} \omega_{m} \mathrm{~d} x=0 \text { for } k \neq m
$$

and $\omega_{k *}>0$ in $(0,1)$, the function $(2.10 \mathrm{a})$ is the only non-negative eigenfunction for (1.3) with $\alpha=\alpha_{*}$ as in (2.9) (more precisely, it is the only eigenfunction that does not change sign in $(0,1)$ ).

Also, since $\omega_{k *}>0$, whenever $y_{0} \geq 0, y_{0} \neq 0$ in $(0,1)$,

$$
\int_{0}^{1} y_{0} \omega_{k *} \mathrm{~d} x>0
$$

(see also Rem. 2.1 below).

Step 4. Moreover,

$$
\left.k *=1 \text { (that is, } \lambda_{1}=0\right) .
$$

Indeed, otherwise (i.e., if $k *>1$ and $\omega_{1}(x) \in H_{0}^{1}(0,1) \subset C[0,1]$ changes sign in $(0,1)$ ) we can select a nonzero non-negative $y_{0}$ such that

$$
\int_{0}^{1} y_{0} \omega_{1} \mathrm{~d} x \neq 0
$$

Therefore, the solution to (1.3) with $\alpha=\alpha_{*}-\lambda_{1}=\alpha_{*}$, according to the (generic) formula (2.2), is represented by the series

$$
\int_{0}^{1} y_{0}(r) \omega_{1}(r) \mathrm{d} r \omega_{1}(x)+\sum_{k=2}^{\infty} \mathrm{e}^{\lambda_{k} t}\left(\int_{0}^{1} y_{0}(r) \omega_{k}(r) \mathrm{d} r\right) \omega_{k}(x),
$$

converging as $t$ increases in $C[0,1]$ to the function (recall that $\lambda_{k}<\lambda_{1}=0$ for $k=2, \ldots$ )

$$
\int_{0}^{1} y_{0}(r) \omega_{1}(r) \mathrm{d} r \omega_{1}(x)
$$

excepting (as we assumed it arguing by contradiction) negative values somewhere in $(0,1)$. This contradicts to the maximum principle (2.7) and hence $(2.10 \mathrm{~b})$ holds.

Step 5. Select now the bilinear control $\alpha$ of the type

$$
\alpha=\alpha_{*}+a, \quad a \in R
$$


Then, the corresponding solution to (1.3) is as follows:

$$
\begin{aligned}
y(x, t) & =\mathrm{e}^{a t} \int_{0}^{1} y_{0}(r) \omega_{1}(r) \mathrm{d} r \omega_{1}(x)+r(x, t) \\
& =\mathrm{e}^{a t} \int_{0}^{1} y_{0}(r) \omega_{1}(r) \mathrm{d} r \omega_{1}(x)+\sum_{k>1} \mathrm{e}^{\left(\lambda_{k}+a\right) t}\left(\int_{0}^{1} y_{0}(r) \omega_{k}(r) \mathrm{d} r\right) \omega_{k}(x),
\end{aligned}
$$

where

$$
\lambda_{k}<0 \text { for } k>1 \text {. }
$$

Accordingly, making use of (2.3) and (2.10a, 2.10b), we have the following estimate:

$$
\begin{aligned}
\left\|y(\cdot, t)-y_{d}\right\|_{L^{2}(0,1)} & \leq\left|\mathrm{e}^{a t} \int_{0}^{1} y_{0} \omega_{1} \mathrm{~d} x-\left\|y_{d}\right\|_{L^{2}(0,1)}\right|+\|r\|_{L^{2}(0,1)} \\
& \leq\left|\mathrm{e}^{a t} \int_{0}^{1} y_{0} \omega_{1} \mathrm{~d} x-\left\|y_{d}\right\|_{L^{2}(0,1)}\right|+\mathrm{e}^{\left(\lambda_{2}+a\right) t}\left\|y_{0}\right\|_{L^{2}(0,1)} .
\end{aligned}
$$

Select now $a$ and $T>0$ such that

$$
\mathrm{e}^{a T} \int_{0}^{1} y_{0} \omega_{1} \mathrm{~d} x=\left\|y_{d}\right\|_{L^{2}(0,1)}
$$

that is,

Then, it follows from (2.13) that

$$
a=\frac{1}{T} \ln \left(\frac{\left\|y_{d}\right\|_{L^{2}(0,1)}}{\int_{0}^{1} y_{0} \omega_{1} \mathrm{~d} x}\right)
$$

$$
\left\|y(\cdot, T)-y_{d}\right\|_{L^{2}(0,1)} \leq \mathrm{e}^{\lambda_{2} T} \frac{\left\|y_{d}\right\|_{L^{2}(0,1)}}{\int_{0}^{1} y_{0} \omega_{1} \mathrm{~d} x}\left\|y_{0}\right\|_{L^{2}(0,1)} \rightarrow 0
$$

as $T$ increases. This ensures (1.4) for some pair $\{a, T\}$ and ends the proof of Theorem 1.1.

Remark 2.1. Given a nonzero non-negative $y_{T}$, in the above argument we used the condition that $y_{0}$ is a nonzero non-negative function only to ensure that the first Fourier coefficient in the solution representation (2.12) is positive. This proves Corollary 1.3 in respect of Theorem 1.1.

\section{Proof of Theorem 1.2: Preliminaries}

To prove Theorem 1.2 we will need the following estimates.

Denote $\mathcal{B}(0, T)=C\left([0, T] ; L^{2}(0,1)\right) \cap H_{0}^{1,0}\left(Q_{T}\right)$ and put

$$
\|q\|_{\mathcal{B}(0, T)}=\left(\max _{t \in[0, T]}\|q(\cdot, t)\|_{L^{2}(0,1)}^{2}+2 \int_{0}^{T} \int_{0}^{1} q_{x}^{2} \mathrm{~d} x \mathrm{~d} s\right)^{1 / 2} .
$$


We have the following a priori estimate:

Lemma 3.1. Given $T>0$ and $\alpha(x) \leq 0$, any solution to (1.1, 1.2a-1.2c) (if there are multiple solutions) satisfies the following two estimates:

$$
\|u\|_{\mathcal{B}(0, T)},\|u\|_{L^{6}\left(Q_{T}\right)} \leq C\left\|u_{0}\right\|_{L^{2}(0,1)} .
$$

The difference $z=u-y$ between any solution $u$ to (1.1, 1.2a-1.2c) (if there are multiple ones) and the unique solution to (1.3) with $y_{0}=u_{0}$ satisfies the following two estimates:

$$
\|z\|_{\mathcal{B}(0, T)},\|z\|_{L^{6}\left(Q_{T}\right)} \leq C\left(T^{\frac{5}{6}\left(1-\frac{r_{1}}{5}\right)}\left\|u_{0}\right\|_{L^{2}(0,1)}^{r_{1}}+T^{\frac{5}{6}\left(1-\frac{3 r_{2}}{5}\right)}\left\|u_{0}\right\|_{L^{2}(0,1)}^{r_{2}}\right) .
$$

Here and below we routinely use symbols $c$ and $C$ to denote (different) generic positive constants.

Proof. Recall [20] that $f\left(\cdot, \cdot, w, w_{x}\right) \in L^{6 / 5}\left(Q_{T}\right)$ and that the following energy equality holds for (1.1) treated as a linear equation with the source term $f\left(x, t, u, u_{x}\right)$, e.g. [20] (p. 142):

$$
\left.\frac{1}{2}\|u\|_{L^{2}(0,1)}^{2}\right|_{0} ^{t}+\int_{0}^{t} \int_{0}^{1}\left(u_{x}^{2}-\alpha u^{2}+f\left(x, s, u, u_{x}\right) u\right) \mathrm{d} x \mathrm{~d} s=0 \quad \forall t \in[0, T] .
$$

Here and everywhere below, if there exist several solutions to (1.1), we always deal separately with one of them at a time, while noticing that all the estimates involved hold uniformly.

Combining (3.3) and (1.2b) and the assumption that $\alpha(x) \leq 0$ immediately yields:

$$
\|u(\cdot, t)\|_{L^{2}(0,1)}^{2}+2 \int_{0}^{t} \int_{0}^{1} u_{x}^{2}(x, s) \mathrm{d} x \mathrm{~d} s \leq\left\|u_{0}\right\|_{L^{2}(0,1)}^{2} \quad \forall t \in[0, T] .
$$

This provides the first estimate in (3.1) with respect to the $\mathcal{B}(0, T)$-norm. The second estimate (with properly arranged generic constant) follows by the continuity of the embedding $\mathcal{B}(0, T)$ into $L^{6}\left(Q_{T}\right)$ (e.g. [20], pp. 467, $75)$ :

$$
\|u\|_{L^{6}\left(Q_{T}\right)} \leq c\|u\|_{\mathcal{B}(0, T)} .
$$

We now intend to evaluate the difference between the solution $u$ to (1.1) with $\alpha(x) \leq 0$ and that to its truncated version (1.3). If $z=u-y$, then

Similar to (3.3) and (3.4) we have for any $\delta>0$,

$$
\begin{gathered}
z_{t}=z_{x x}+\alpha z-f\left(x, t, u, u_{x}\right) \quad \text { in } Q_{T}, \\
\left.z\right|_{x=0,1}=0,\left.\quad z\right|_{t=0}=0 .
\end{gathered}
$$

$$
\begin{gathered}
\|z(\cdot, t)\|_{L^{2}(0,1)}^{2}+2 \int_{0}^{t} \int_{0}^{1} z_{x}^{2}(x, s) \mathrm{d} x \mathrm{~d} s \leq-2 \int_{0}^{t} \int_{0}^{1} z f\left(x, s, u, u_{x}\right) \mathrm{d} x \mathrm{~d} s \\
\leq 2\|z\|_{L^{6}\left(Q_{t}\right)}\left\|f\left(\cdot, \cdot, u, u_{x}\right)\right\|_{L^{6 / 5}\left(Q_{t}\right)} \leq 2 c\|z\|_{\mathcal{B}(0, t)}\left\|f\left(\cdot, \cdot, u, u_{x}\right)\right\|_{L^{6 / 5}\left(Q_{T}\right)} \\
\leq \delta\|z\|_{\mathcal{B}(0, t)}^{2}+\frac{c^{2}}{\delta}\left\|f\left(\cdot, \cdot, u, u_{x}\right)\right\|_{L^{6 / 5}\left(Q_{T}\right)}^{2} \quad \forall t \in[0, T],
\end{gathered}
$$

where we have used Hölder's and Young's inequalities and, again, equation (3.5). 
From (3.6), we have

$$
\max _{\tau \in(0, t)}\|z(\cdot, \tau)\|_{L^{2}(0,1)}^{2} \leq \delta\|z\|_{\mathcal{B}(0, t)}^{2}+\frac{c^{2}}{\delta}\left\|f\left(\cdot, \cdot, u, u_{x}\right)\right\|_{L^{6 / 5}\left(Q_{T}\right)}^{2} \quad \forall t \in[0, T] .
$$

Hence, again from (3.6),

$$
\|z\|_{\mathcal{B}(0, t)}^{2} \leq 2 \delta\|z\|_{\mathcal{B}(0, t)}^{2}+\frac{2 c^{2}}{\delta}\left\|f\left(\cdot, \cdot, u, u_{x}\right)\right\|_{L^{6 / 5}\left(Q_{T}\right)}^{2} \quad \forall t \in[0, T]
$$

and

or

$$
(1-2 \delta)\|z\|_{\mathcal{B}(0, t)}^{2} \leq \frac{2 c^{2}}{\delta}\left\|f\left(\cdot, \cdot, u, u_{x}\right)\right\|_{L^{6 / 5}\left(Q_{T}\right)}^{2}
$$

$$
\|z\|_{\mathcal{B}(0, T)} \leq \frac{\sqrt{2} c}{\sqrt{\delta(1-2 \delta)}}\left\|f\left(\cdot, \cdot, u, u_{x}\right)\right\|_{L^{6 / 5}\left(Q_{T}\right)}
$$

provided that

$$
0<\delta<\frac{1}{2}
$$

Now, using (1.2a) and Hölder's inequality (as in [16], p. 469; and [10], p. 863), we obtain:

$$
\left\|f\left(\cdot, \cdot, u, u_{x}\right)\right\|_{L^{6 / 5}\left(Q_{T}\right)} \leq \beta T^{\frac{5}{6}\left(1-\frac{r_{1}}{5}\right)}\|u\|_{L^{6}\left(Q_{T}\right)}^{r_{1}}+\beta T^{\frac{5}{6}\left(1-\frac{3 r_{2}}{5}\right)}\left\|u_{x}\right\|_{L^{2}\left(Q_{T}\right)}^{r_{2}} .
$$

Combining $(3.8,3.9)$ with (3.1) and, again, with (3.5) yields (3.2). This completes the proof of Lemma 3.1.

Remark 3.1. Remark 3.1. In [16] we derived the estimates of type (3.1) and (3.2) (in somewhat more general situation) for the case when $\alpha$ is a positive constant. They are as follows:

$$
\begin{gathered}
\|u\|_{\mathcal{B}(0, T)},\|u\|_{L^{6}\left(Q_{T}\right)} \leq C \mathrm{e}^{\alpha T}\left\|u_{0}\right\|_{L^{2}(0,1)}, \\
\|z\|_{\mathcal{B}(0, T)},\|z\|_{L^{6}\left(Q_{T}\right)} \leq C \mathrm{e}^{\frac{2 \alpha T}{1-\delta}} \frac{1}{\sqrt{\delta}}\left(T^{\frac{5}{6}\left(1-\frac{r_{1}}{5}\right)}\|u\|_{L^{6}\left(Q_{T}\right)}^{r_{1}}\right. \\
\left.+T^{\frac{5}{6}\left(1-\frac{3 r_{2}}{5}\right)}\left\|u_{x}\right\|_{L^{2}\left(Q_{T}\right)}^{r_{2}}\right) \quad \forall \delta \in(0,1 / 2),
\end{gathered}
$$

where $C$ does not depend on $\alpha$. We will also use them in the proof of Theorem 1.2 below.

\section{Proof of Theorem 1.2}

In [16] we proved the global approximate controllability in $L^{2}(0,1)$ at any time $T>0$ of the following Dirichlet boundary problem

$$
\begin{gathered}
w_{t}=w_{x x}+k(t) w-f\left(x, t, w, w_{x}\right)+v(t) \chi_{\left(l_{1}, l_{2}\right)}(x) \text { in } Q_{T}, \\
\left.w\right|_{x=0,1}=0,\left.\quad w\right|_{t=0}=w_{0} \in L^{2}(0,1), \quad k \in L^{\infty}(0, T), v \in L^{2}(0, T),
\end{gathered}
$$

governed by the combination of two controls - the bilinear lumped piecewise constant control $k=k(t)$ and the additive lumped control $v(t) \chi_{\left(l_{1}, l_{2}\right)}(x)$ supported in the given subinterval $\left(l_{1}, l_{2}\right) \subset(0,1)\left(l_{1} \pm l_{2}\right.$ are irrational), also assuming that conditions $(1.2 \mathrm{a}-1.2 \mathrm{c})$ hold. (In [16] the case of several dimensions was treated as well with the locally distributed controls in place of the lumped additive ones.)

More precisely, it was noticed in [16] that one can steer a system like (4.1) with $v=0$ in $L^{2}(\Omega)$ from any initial state $w_{0}$ arbitrarily close to any state like $s_{*} w_{0}$ for any $s_{*}>0$, given in advance, by applying "suitably large" constant bilinear controls for a "very short" time. The global controllability result in [16] then follows by combining this property with available "quasi-local" controllability of the system at hand when it is governed 
solely by means of the additive controls of localized support, namely, along the following scheme:

- given the initial and target states $w_{0}$ and $w_{d}$, we steer (4.1) "close enough" to the zero state (equilibrium), while employing a suitable constant negative bilinear control $k$ only;

- using (a sort of) locally controllability technique with only additive controls $v$ active, we then steer (4.1) to a state $s_{*} w_{d}+o\left(s_{*}\right)$ for some small parameter $s_{*}>0$;

- again, employing only a suitable constant positive bilinear control $k$, we "stretch" the latter state to the desirable target state $w_{d}$.

As one can see, the additive controls play the most crucial role to achieve the principal "fine" steering in the above scheme.

Now note that if we manage to replace them somehow by (principally different) bilinear static controls, then the above scheme can be used to prove Theorem 1.2 in the framework of the non-negative controllability (recall that the traditional approximate controllability never holds by means of bilinear controls, as we discussed it in the introduction). This replacement is the crux of our proof below.

We intend to modify the first two of the above three steps, making use of Theorem 1.1 and Lemma 3.1. Our goal will be to steer system $(1.1,1.2 \mathrm{a}-1.2 \mathrm{c})$ from $u_{0}$ to a state $s_{*} u_{d}+o\left(s_{*}\right)$ for some small parameter $s_{*}>0$. To this end, we will make use of the "superlinearity" assumptions (1.2a-1.2c), which ensure that the system at hand behaves "almost" like the linear one near the origin. Then the last step from the above scheme applies.

Proof. of Theorem 1.2. Fix any non-negative $u_{0} \neq 0$ in $L^{2}(0,1)$ and $u_{d}$ satisfying the assumptions on $y_{d}$ described in (2.8).

Step 1. Select any $t_{*}>0$. On the interval $\left(0, t_{*}\right)$ we intend to apply a negative constant control $\alpha(x)=\lambda$ (its value will be chosen later). Then for the corresponding solution $y$ to (1.3) with $y_{0}=u_{0}$ we have (using the generic representation $(2.2))$ :

$$
y\left(x, t_{*}\right)=\mathrm{e}^{\lambda t_{*}} \sum_{k=1}^{\infty} \mathrm{e}^{\lambda_{k} t_{*}}\left(\int_{0}^{1} y_{0}(r) \omega_{k}(r) \mathrm{d} r\right) \omega_{k}(x),
$$

where in this case $\lambda_{k}=-(\pi k)^{2}, \omega_{k}(x)=\sqrt{2} \sin \pi k x$.

Fix any $s \in(0,1)$ and select now (a constant on $\left(0, t_{*}\right)$ bilinear control) $\lambda=\lambda\left(t_{*}, s\right)<0$ such that

$$
\mathrm{e}^{\lambda t_{*}}=s
$$

Then, it follows from (4.2) that

$$
y\left(\cdot, t_{*}\right) \rightarrow s y_{0}(\cdot)=s u_{0} \text { as } t_{*} \rightarrow 0+.
$$

Furthermore, using the estimates (3.2) we also obtain the same convergence for the corresponding solution to (1.1) (uniform over all the possible multiple solutions). In other words, we can steer, arbitrarily fast, (1.1) from any initial state $u_{0}=y_{0}$ to a state

$$
u\left(\cdot, t_{*}\right)=s y_{0}+o(s)=s u_{0}+o(s) \quad \text { as } s \rightarrow 0+
$$

for some $t_{*}=t_{*}(s)$, where

$$
\|o(s) / s\|_{L^{2}(0,1)} \rightarrow 0 \text { as } s \rightarrow 0 .
$$

We further assume that $s$ is "small" (to ensure (4.3) and (4.4) whenever it will be necessary).

Step 2. Select

$$
\alpha_{*}(x)=-\frac{u_{d x x}(x)}{u_{d}(x)}
$$


as in (2.9). The corresponding first eigenfunction for the parabolic operator in (1.3) (see (2.10a, 2.10b)) will be

$$
\frac{u_{d}(x)}{\left\|u_{d}\right\|_{L^{2}(0,1)}}=\omega_{1}(x)
$$

We now apply Theorem 1.1 for the linear system (1.3) on some interval $\left(t_{*}, t^{*}\right)$, where $t^{*}$ will be selected later, with

$$
s u_{0}+o(s)
$$

from (4.3) in place of the initial state (note that for small $s$ Rem. 2.1 applies - this will eventually yield Cor. 1.3 for Th. 1.2) and with

$$
s^{1+\xi} u_{d}, \text { where } \xi \in\left(0, \min \left\{r_{1}, r_{2}\right\}-1\right)
$$

$\left(r_{1}\right.$ and $r_{2}$ are from $(1.2 \mathrm{a}-1.2 \mathrm{c})$ ), in place of the target state. Accordingly (along the lines (2.12-2.14) and using (4.5) and (4.6)), we will have the estimate as in (2.14):

$$
\left\|y\left(\cdot, t^{*}\right)-s^{1+\xi} u_{d}\right\|_{L^{2}(0,1)} \leq \mathrm{e}^{\lambda_{2}\left(t^{*}-t_{*}\right)} \frac{s^{1+\xi}\left\|u_{d}\right\|_{L^{2}(0,1)}}{\int_{0}^{1}\left(s y_{0}+o(s)\right) \omega_{1} \mathrm{~d} x}\left\|s y_{0}+o(s)\right\|_{L^{2}(0,1)}
$$

for some $t^{*}>t_{*}$ (where $\lambda_{k}$ 's are the eigenvalues associated with $\alpha_{*}, \lambda_{1}=0$ ). Here, as in Step 5 of the proof of Theorem 1.1, $y$ is the solution to (1.3) on $\left(t_{*}, t^{*}\right)$ with bilinear control $\alpha(x)=\alpha_{*}(x)+a$ such that

$$
\mathrm{e}^{a\left(t^{*}-t_{*}\right)} \int_{0}^{1}\left(s y_{0}+o(s)\right) \omega_{1} \mathrm{~d} x=s^{1+\xi}\left\|u_{d}\right\|_{L^{2}(0,1)}
$$

or, whenever $a \neq 0$,

$$
t^{*}=t_{*}+\frac{1}{a} \ln \left(\frac{s^{1+\xi}\left\|u_{d}\right\|_{L^{2}(0,1)}}{\int_{0}^{1}\left(s y_{0}+o(s)\right) \omega_{1} \mathrm{~d} x},\right)
$$

and $\lambda_{1}$ and $\lambda_{2}$ are the first two eigenvalues for (1.3) with $\alpha=\alpha_{*}$.

Select now

where $\rho>0$ is some (fixed) constant. Since $\lambda_{1}=0$,

$$
a=-\left\|\alpha_{*}\right\|_{L^{\infty}(0,1)}-\rho,
$$

$$
a<0 \quad \text { and } \quad \alpha(x)=\alpha_{*}(x)+a<0, \quad x \in[0,1] .
$$

Hence, by (4.8),

and also the estimate (3.2) applies on the interval $\left(t_{*}, t^{*}\right)$ :

$$
t^{*}-t_{*}=t^{*}(s)-t_{*}(s) \rightarrow \infty \quad \text { as } s \rightarrow 0+
$$

$$
\begin{gathered}
\left\|u\left(\cdot, t^{*}\right)-s^{1+\xi} u_{d}\right\|_{L^{2}(0,1)} \leq\left\|u\left(\cdot, t^{*}\right)-y\left(\cdot, t^{*}\right)\right\|_{L^{2}(0,1)}+\left\|y\left(\cdot, t^{*}\right)-s^{1+\xi} u_{d}\right\|_{L^{2}(0,1)} \\
\leq C\left(t^{*}-t_{*}\right)^{\max \left\{\frac{5}{6}\left(1-\frac{r_{1}}{5}\right), \frac{5}{6}\left(1-\frac{3 r_{2}}{5}\right)\right\}} s^{\min \left\{r_{1}, r_{2}\right\}}+\left(C s^{\xi \lambda_{2} / a}\right) s^{1+\xi}\left\|u_{d}\right\|_{L^{2}(0,1)} \\
=o\left(s^{1+\xi}\right) \text { as } s \rightarrow 0+
\end{gathered}
$$

(we remind the reader that $C$ denotes a generic positive constant). Here we also used (4.7) and (4.9) to show that as $s \rightarrow 0+$ :

$$
\mathrm{e}^{\lambda_{2}\left(t^{*}-t_{*}\right)}=\left(\frac{s^{1+\xi}\left\|u_{d}\right\|_{L^{2}(0,1)}}{\int_{0}^{1}\left(s y_{0}+o(s)\right) \omega_{1} \mathrm{~d} x}\right)^{\frac{\lambda_{2}}{a}} \leq C s^{\xi \lambda_{2} / a}
$$


(with $\lambda_{2}<\lambda_{1}=0$ and $a$ as in (4.9)) and that

$$
\begin{gathered}
\left(t^{*}-t_{*}\right)^{\max \left\{\frac{5}{6}\left(1-\frac{r_{1}}{5}\right), \frac{5}{6}\left(1-\frac{3 r_{2}}{5}\right)\right\}} s^{\min \left\{r_{1}, r_{2}\right\}} \\
=\left(\frac{1}{a} \ln \left(\frac{s^{1+\xi}\left\|u_{d}\right\|_{L^{2}(0,1)}}{\int_{0}^{1}\left(s y_{0}+o(s)\right) \omega_{1} \mathrm{~d} x}\right)\right)^{\max \left\{\frac{5}{6}\left(1-\frac{r_{1}}{5}\right), \frac{5}{6}\left(1-\frac{3 r_{2}}{5}\right)\right\}} s^{\min \left\{r_{1}, r_{2}\right\}} \\
\leq\left(C|\ln s|^{\max \left\{\frac{5}{6}\left(1-\frac{r_{1}}{5}\right), \frac{5}{6}\left(1-\frac{3 r_{2}}{5}\right)\right\}} s^{\min \left\{r_{1}, r_{2}\right\}-1-\xi}\right) s^{1+\xi},
\end{gathered}
$$

also recalling that $r_{1}, r_{2}>1$ in $(1.2 \mathrm{a}-1.2 \mathrm{c})$ and $\xi \in\left(0, \min \left\{r_{1}, r_{2}\right\}-1\right)$.

Thus, we showed that

$$
u\left(\cdot, t^{*}\right)=s^{1+\xi} u_{d}+o\left(s^{1+\xi}\right) \text { as } s \rightarrow 0+.
$$

Remark 4.1. We would like to point out here that the "superlinearity" condition (1.2c) is essential in the above to ensure that $\min \left\{r_{1}, r_{2}\right\}>1$.

Step 3. Now we apply the argument in the last step of the proof in [16] as outlined in the beginning of this section with $s_{*}=s^{1+\xi}$. Namely, on some interval $\left(t^{*}, T\right)$ (with $T$ to be selected later) we apply positive constant control

$$
\alpha(x) \equiv \alpha>0, \quad t \in\left(t^{*}, T\right)
$$

In the fashion of Steps 1 and 2 in the above, but based on the estimates (3.10) and (3.11) in Remark 3.1 in place of Lemma 3.1, it was shown in [16] that with parameters $\varepsilon=T-t^{*}=T-t^{*}(s), s_{*}=s^{1+\xi}$, and $\alpha=\alpha\left(\varepsilon, s_{*}\right)$, selected so that

(A) $s_{*} \rightarrow 0+$;

(B) $\mathrm{e}^{\alpha \varepsilon}=\frac{1}{s_{*}}$

(C) $\varepsilon \rightarrow 0$, so that

$$
\mathrm{e}^{\frac{2 \alpha \varepsilon}{1-\delta}} \varepsilon^{\min \left\{\frac{5}{6}\left(1-\frac{r_{1}}{5}\right) ; \frac{5}{6}\left(1-\frac{3 r_{2}}{5}\right)\right\}}=\varepsilon^{\min \left\{\frac{5}{6}\left(1-\frac{r_{1}}{5}\right) ; \frac{5}{6}\left(1-\frac{3 r_{2}}{5}\right)\right\}} s_{*}^{-\frac{2}{1-\delta}} \rightarrow 0
$$

we will have for some $T>0$ that

$$
\left\|u(\cdot, T)-u_{d}\right\|_{L^{2}(0,1)} \rightarrow 0 .
$$

This completes the proof of Theorem 1.2.

\section{Concluding Remarks}

It is worth noticing that, in terms of applications, the method used to prove our main linear result Theorem 1.1- allows one to deal with relatively small and simple (and hence "practical") static controls, which, however, may need to be applied for a relatively long time. (These controls were also used in the crucial part of the proof of Th. 1.3.) On the other hand, this method requires the first eigenvalues be always simple, which was achieved by assuming that $n=1$.

Accordingly, with the changes necessary to take care about the regularity of solutions to $(1.1,1.3)$ and the approximation procedure described in Step 2 of the proof of Theorem 1.1, the arguments of Theorem 1.1 and 1.2 may apply in the general $n$-dimensional case as well, provided that the first eigenvalue of $(1.1,1.3)$ associated with $\alpha_{*}$ as in (2.9) is simple. In other words, the corresponding $n$-dimensional version of systems (1.3) and (1.1) can be steered from any nonzero non-negative initial state arbitrarily close to any of the non-negative target states generating such $\alpha_{*}$ 's.

In the recent work [18] (written after the current paper was submitted) we obtained a different non-negative controllability result for a system like in (1.1) in several space dimensions with the terms $f$ which can be superlinear at infinity but are not necessarily superlinear near the origin. The result in [18] always requires 
at least three "large" static bilinear controls (whose magnitude increases as the precision of steering increases) applied subsequently for very short times. Unlike the present paper, based on the use of the dynamics imposed by the diffusion-reaction term like $y_{x x}+\alpha(x) y$ in (1.3) in the first place, the method of [18] focuses on the "suppression" of the effect of the diffusion term like the above-mentioned $y_{x x}$. It makes use of the part of the dynamics of (1.1) which can be approximated by the trajectories of the ordinary differential equation $d z / d t=\alpha(x) z$ in $L^{2}(\Omega)$. Accordingly, the method of [18] does not apply to obtain the results of Theorem 1.1, allowing the use of "small" single static controls, and of Corollary 1.3, allowing the principal extension of the set of non-negative initial states to the set described in conditions (1.5).

\section{Additional related references:}

- The works [17] and [10] (which also appeared after the present paper was submitted) deal with a different approach to the bilinear controllability, which is as follows. It is known that the heat equation is approximately controllable in $L^{2}(\Omega)$ by the additive static controls $v=v(x)$ with support everywhere in the space domain $\Omega$. Then a suitable bilinear control for (1.3) can be sought as some "well-posed modification" of the expression $\alpha(x, t)=v(x) / y(x, t)$, in which case the "original" additive static control $v(x)$ is artificially "transformed" into the bilinear term $\alpha(x, t) y(x, t)$. Note however, that this approach deals with essentially more "complex" controls (e.g., in terms of practical implementation), namely, as functions of both $x$ and $t$. In the paper [10] it was applied in the context of the non-negative approximate controllability for a special class of the semilinear parabolic equations whose solutions satisfy the maximum principle. In [17] it was used to investigate the exact null-controllability of a semilinear nonhomogeneous version of (1.3) with the bilinear term like $\alpha(x, t)(f(x, t, y)-\theta(x))$, where $f$ is sublinear and $\theta$ is given. (Note that the exact null-controllability discussed in [17] is out of question for the homogeneous bilinear system like (1.1) (or (1.3)), since the origin is always a fixed point for such a system for any control $\alpha$.)

- We also would like to mention the works $[7,21]$ (and the references therein) on the issue of optimal bilinear control for various pde's.

- An extensive and thorough bibliography on controllability on bilinear ode's is available, see, e.g., the survey [2]. The research in this area was originated in the $60 \mathrm{~s}$, on the one hand, by the works of Kucera, who linked this area to Lie Algebra approach, and, on the other hand, by the works of Mohler, who pursued the qualitative approach and numerous applications.

- A side from controllability, a very close issue is stabilization by means of bilinear controls. Again, we can point out only at a very limited publications in this area in terms of pde's, see [3,4,22].

\section{REFERENCES}

[1] S. Anita and V. Barbu, Null controllability of nonlinear convective heat equations. ESAIM: COCV 5 (2000) 157-173.

[2] A. Baciotti, Local Stabilizability of Nonlinear Control Systems. World Scientific, Singapore, Series on Advances in Mathematics and Applied Sciences 8 (1992).

[3] J.M. Ball and M. Slemrod, Feedback stabilization of semilinear control systems. Appl. Math. Opt. 5 (1979) 169-179.

[4] J.M. Ball and M. Slemrod, Nonharmonic Fourier series and the stabilization of distributed semi-linear control systems. Comm. Pure. Appl. Math. 32 (1979) 555-587.

[5] J.M. Ball, J.E. Mardsen and M. Slemrod, Controllability for distributed bilinear systems. SIAM J. Control Optim. (1982) $575-597$.

[6] V. Barbu, Exact controllability of the superlinear heat equation. Appl. Math. Opt. 42 (2000) 73-89.

[7] M.E. Bradley, S. Lenhart and J. Yong, Bilinear optimal control of the velocity term in a Kirchhoff plate equation. J. Math. Anal. Appl. 238 (1999) 451-467.

[8] E. Fernández-Cara, Null controllability of the semilinear heat equation. ESAIM: COCV 2 (1997) 87-103.

[9] E. Fernández-Cara and E. Zuazua, Controllability for blowing up semilinear parabolic equations. C. R. Acad. Sci. Paris Sér. I Math. 330 (2000) 199-204.

[10] L.A. Fernández, Controllability of some semilnear parabolic problems with multiplicativee control, a talk presented at the Fifth SIAM Conference on Control and its applications, held in San Diego, July 11-14, 2001 (in preparation).

[11] A. Fursikov and O. Imanuvilov, Controllability of evolution equations. Res. Inst. Math., GARC, Seoul National University, Lecture Note Ser. 34 (1996). 
[12] J. Henry, Étude de la contrôlabilité de certaines équations paraboliques non linéaires, Thèse d'état. Université Paris VI (1978).

[13] A.Y. Khapalov, Approximate controllability and its well-posedness for the semilinear reaction-diffusion equation with internal lumped controls. ESAIM: COCV 4 (1999) 83-98.

[14] A.Y. Khapalov, Global approximate controllability properties for the semilinear heat equation with superlinear term. Rev. Mat. Complut. 12 (1999) 511-535.

[15] A.Y. Khapalov, A class of globally controllable semilinear heat equations with superlinear terms. J. Math. Anal. Appl. 242 (2000) 271-283.

[16] A.Y. Khapalov, Bilinear control for global controllability of the semilinear parabolic equations with superlinear terms, in the Special volume "Control of Nonlinear Distributed Parameter Systems", dedicated to David Russell, Marcel Dekker, Vol. 218 (2001) 139-155.

[17] A.Y. Khapalov, On bilinear controllability of the parabolic equation with the reaction-diffusion term satisfying Newton's Law, in the special issue of the J. Comput. Appl. Math. dedicated to the memory of J.-L. Lions (to appear).

[18] A.Y. Khapalov, Controllability of the semilinear parabolic equation governed by a multiplicative control in the reaction term: A qualitative approach, Available as Tech. Rep. 01-7, Washington State University, Department of Mathematics (submitted).

[19] K. Kime, Simultaneous control of a rod equation and a simple Schrödinger equation. Systems Control Lett. 24 (1995) $301-306$.

[20] O.H. Ladyzhenskaya, V.A. Solonikov and N.N. Ural'ceva, Linear and Quasi-linear Equations of Parabolic Type. AMS, Providence, Rhode Island (1968).

[21] S. Lenhart, Optimal control of convective-diffusive fluid problem. Math. Models Methods Appl. Sci. 5 (1995) $225-237$.

[22] S. Müller, Strong convergence and arbitrarily slow decay of energy for a class of bilinear control problems. J. Differential Equations 81 (1989) 50-67. 